University of Nebraska - Lincoln

DigitalCommons@University of Nebraska - Lincoln

2017

Ferroelectricity and the phase transition in large area evaporated vinylidene fluoride oligomer thin films

Keith Foreman

Shashi Poddar

Stephen Ducharme

Shireen Adenwalla

Follow this and additional works at: https://digitalcommons.unl.edu/physicsadenwalla

Part of the Condensed Matter Physics Commons, Engineering Physics Commons, Materials Science and Engineering Commons, and the Nanoscience and Nanotechnology Commons

This Article is brought to you for free and open access by the Research Papers in Physics and Astronomy at DigitalCommons@University of Nebraska - Lincoln. It has been accepted for inclusion in Shireen Adenwalla Papers by an authorized administrator of DigitalCommons@University of Nebraska - Lincoln. 


\title{
Ferroelectricity and the phase transition in large area evaporated vinylidene fluoride oligomer thin films
}

\author{
K. Foreman, ${ }^{1,2, a)}$ Shashi Poddar, ${ }^{1,2,3}$ Stephen Ducharme, ${ }^{1,2}$ and S. Adenwalla ${ }^{1,2}$ \\ ${ }^{1}$ Department of Physics and Astronomy, University of Nebraska-Lincoln, Lincoln, NE 68588-0299, USA \\ ${ }^{2}$ Nebraska Center for Materials and Nanoscience, University of Nebraska-Lincoln, Lincoln, NE 68588-0298, \\ USA \\ ${ }^{3}$ Université de Louvain, Bio and Soft Matter, Institute of Condensed Matter and Nanoscience, \\ Louvain-la-Neuve, Belgium
}

(Received 20 March 2017; accepted 8 May 2017; published online 19 May 2017)

\begin{abstract}
Organic ferroelectric materials, including the well-known poly(vinylidene fluoride) and its copolymers, have been extensively studied and used for a variety of applications. In contrast, the VDF oligomer has not been thoroughly investigated and is not widely used, if used at all. One key advantage the oligomer has over the polymer is that it can be thermally evaporated in vacuum, allowing for the growth of complex heterostructures while maintaining interfacial cleanliness. Here, we report on the ferroelectric properties of high-quality VDF oligomer thin films over relatively large areas on the order of $\mathrm{mm}^{2}$. The operating temperature is identified via differential scanning calorimetry and pyroelectric measurements. Pyroelectric measurements also reveal a stable remanent polarization for these films which persists over very long time scales, an important result for nonvolatile data storage. Temperature dependent pyroelectric and capacitance measurements provide compelling evidence for the phase transition in these films. Capacitance-voltage and currentvoltage measurements are used to confirm ferroelectricity, quantify the dielectric loss, and calculate the spontaneous polarization. Finally, piezoresponse force microscopy is used to demonstrate large area, low-voltage ferroelectric domain reading/writing in VDF oligomer thin films. This work enables new channels for VDF oligomer applications and research. Published by AIP Publishing.

[http://dx.doi.org/10.1063/1.4983820]
\end{abstract}

\section{INTRODUCTION}

Organic molecules can be selected, or designed, to suit specific needs, ${ }^{1}$ leading to a rapid increase in both their use in fundamental physics and materials investigations, as well as in device applications. The latter includes organic light emitting diode (OLED) displays, ${ }^{2-4}$ flexible electronics, ${ }^{5-7}$ and even potential applications using voltage-controlled magnetic anisotropy. ${ }^{8-10}$ Organic ferroelectric materials effectively combine the customizability afforded by organic synthesis with the useful electro-physical behaviors of ferroelectric materials, applications of which range from piezoelectric transducers ${ }^{11}$ to data storage. ${ }^{5,12,13}$ The well-known polymer ferroelectric poly(vinylidene fluoride) $(\mathrm{PVDF})^{14}$ and its copolymers with trifluoroethylene (TrFE) have several properties that make them well-suited for various applications, including a low stiffness coefficient ${ }^{15}$ and a high remanent polarization. ${ }^{16}$ As with other organic materials, $\mathrm{P}(\mathrm{VDF}-\mathrm{TrFE})$ can also be customized to suit specific applications. For example, the transition temperature may be modified by tuning the ratio of VDF to TrFE. ${ }^{17}$

Here, we report an investigation of ferroelectricity in a promising organic material, the VDF oligomer. Obtaining definitive experimental evidence of ferroelectricity in new materials is an intricate process since non-ferroelectric materials may mimic ferroelectric signatures. ${ }^{18}$ For example, charge injection in an electret can result in ferroelectric-like

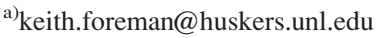

hysteresis loops, with very long relaxation times. We confirm the existence of ferroelectricity using a variety of measurements. More importantly, however, we present compelling evidence of the existence of the ferroelectric-to-paraelectric phase transition, which has not been previously identified in VDF oligomer crystals.

The VDF oligomer shares the same molecular and crystal structure as the polymer counterpart, PVDF. ${ }^{17,19,20}$ This similarity, in turn, may explain the similar ferroelectric properties of the VDF oligomer and the polymer. However, the VDF oligomer holds a crucial advantage over the polymer: VDF oligomer thin films can be deposited in vacuum. Unlike PVDF, which is commonly deposited in ambient conditions using Langmuir-Blodgett deposition ${ }^{21}$ or spin coating, ${ }^{22}$ vacuum deposition of the VDF oligomer preserves the cleanliness of the interface between the organic and adjacent layers, ${ }^{23}$ an essential need for spintronics and other devices. Our earlier work ${ }^{23-25}$ has established the conditions for optimal growth via thermal evaporation, investigated the physical properties of these thermally evaporated VDF oligomer thin films, and established that these thin films both protect and are chemically inert with an underlying metal thin film, thereby preserving the interface.

Because of the potential device applications of VDF oligomer thin films, we also investigate those properties relevant to devices, including the working temperature range and remanent polarization, the reliability and reproducibility of switching, and the time dependence of the remanent polarization. The latter is especially important for non-volatile 
memory applications. We establish the working temperature of VDF oligomer thin films and provide evidence for the existence of the ferroelectric-to-paraelectric phase transition using a combination of differential scanning calorimetry along with pyroelectric and capacitance measurements as a function of substrate temperature. The measurements of the dependence of capacitance on bias voltage elucidate the ferroelectric nature of the VDF oligomer and demonstrate the repeatability of polarization switching in these films. The loss tangent of VDF oligomer films is recorded as a function of voltage, revealing that these films maintain low ac dielectric loss even during polarization reversal. Current-voltage measurements (I-V) are used to calculate the spontaneous polarization of these VDF oligomer films. Moreover, the capacitance, loss tangent, and I-V measurements were recorded from VDF oligomer films with areas over $2 \mathrm{~mm}^{2}$, showing that high-quality films can be produced on scales suitable for devices. X-ray diffraction (XRD) measurements are used to assess the ferroelectric structure of as-grown VDF oligomer thin films. Measurements of the zero-field pyroelectric signal as a function of time indicate a stable, long-lived remanent polarization, a crucial result for device applications. Finally, using piezoresponse force microscopy (PFM), large area ferroelectric domain writing is demonstrated, opening the way to possible memory applications.

\section{SAMPLE PREPARATION AND EXPERIMENTAL METHODS}

Samples were prepared in several configurations to suit particular measurements. Specific sample structures are listed below with each corresponding measurement, and the sample architectures are shown in the insets of the accompanying figures. Substrates were either glass slides or $\mathrm{Si}$ wafers. For samples requiring a bottom metallic electrode, Pt thin films $(30-50 \mathrm{~nm})$ were deposited at a rate of $0.5 \AA / \mathrm{s}$ in a magnetron sputtering deposition chamber with a base pressure of $1 \times 10^{-8}$ Torr. For samples not requiring metallic electrodes, the VDF oligomer was deposited directly onto the substrate. The VDF oligomer thin films were deposited in a custom thermal evaporation chamber connected to the sputtering chamber via a gate valve. ${ }^{24}$ The substrate temperature was maintained at $130 \mathrm{~K}$ during the deposition to ensure that the VDF oligomer chain conformation was in the desired ferroelectric $\beta$-phase. ${ }^{26}$ The VDF oligomer was deposited at a rate of $1 \AA / s$, as confirmed by ellipsometry measurements. ${ }^{25}$ The VDF oligomer powder, $\mathrm{CF}_{3}-\left(\mathrm{CH}_{2} \mathrm{CF}_{2}\right)_{\mathrm{n}}-\mathrm{I}$, was provided by Kunshan Hisense Electronic Co., Ltd. and has a chain length of $n=15 \pm 2$ as confirmed by nuclear magnetic resonance measurements. For samples requiring a top electrode, 30-nm thick $\mathrm{Al}$ films were deposited at a rate of $2 \AA / s$ from a thermal evaporator at a pressure of $3.75 \times 10^{-5}$ Torr. Thermal evaporation was used to deposit top metal electrodes, as sputtering was found to be unsuitable due to the highly energetic sputtered atoms which puncture the soft organic film, resulting in electrically shorted top and bottom electrodes.

The absorption and release of latent heat of a ferroelectric being driven through its phase transition are manifested as peaks in heat flow vs. temperature measurements using differential scanning calorimetry. VDF oligomer powder was loaded into an alumina pan of a NETZSCH Model 204 F1 Phoenix calorimeter, sealed, and placed in the calorimetry chamber along with an empty reference alumina pan. The calorimetry chamber was purged with argon gas to avoid contamination, and the heating/cooling rate was maintained at $2{ }^{\circ} \mathrm{C} / \mathrm{min}$.

The pyroelectric current, which is proportional to the spontaneous polarization, ${ }^{27,28}$ was measured using the Chynoweth method ${ }^{29}$ (described in more detail in the following section), with a $1-\mathrm{mW}, 658-\mathrm{nm}$ wavelength diode laser modulated with an optical chopper at a frequency of $2 \mathrm{kHz}$. A thermoelectric heater was integrated into the experimental setup so that the pyroelectric current could be measured as a function of sample temperature. XRD measurements were performed using a Rigaku D/Max-B Diffractometer in the $\theta-2 \theta$ mode with $\mathrm{Co} \mathrm{K} \alpha$ radiation of wavelength $1.789 \AA$.

The dependence of the capacitance and loss tangent on voltage $(\mathrm{C}-\mathrm{V})$ were measured using a programmable HP 4192A LF impedance analyzer with an ac test signal of $0.1 \mathrm{~V}$ at $1.0 \mathrm{kHz}$. A LabVIEW-controlled thermoelectric heater was used to heat the sample in order to measure the capacitance as a function of temperature. The static I-V characteristic curves were measured for the VDF oligomer capacitors using a LabVIEW-controlled Keithley Model 2400 source meter. From the I-V curve, the value of the remanent polarization can be calculated. ${ }^{20}$ High-resolution optical microscopy images along with ImageJ software ${ }^{30}$ were used to measure the electrode area for these calculations.

Local domain imaging of ferroelectric VDF oligomer thin films was performed by PFM using a Bruker Dimension Icon atomic force microscope in the piezoresponse mode, which is optimal for visualizing and manipulating vertical, out-of-plane domains. For these studies, a 30-nm layer of Pt was sputtered onto a Si substrate, followed by the deposition of a VDF oligomer thin film. A conductive Pt-Ir coated PFM tip (Bruker model SCM-PIC-V2) with a nominal spring constant of $0.1 \mathrm{~N} / \mathrm{m}$ was used for reading and writing ferroelectric domains. The soft organic films are bound by weak van der Waals forces, ${ }^{1,23}$ necessitating the relatively soft PFM tip to ensure that there are no topographical changes in the surface of the soft film during PFM measurements without compromising the piezoresponse signal. An ac voltage at a frequency of $45 \mathrm{kHz}$ is applied between the tip and sample during domain reading/mapping. The writing of rectangular domains is accomplished by scanning the film surface with a dc bias up to $\pm 10 \mathrm{~V}$ applied between the tip and the sample. In both cases, the platinum-coated silicon substrate acts as the ground.

\section{RESULTS AND DISCUSSION}

Although the observation of bi-stable polarization hysteresis adequately defines ferroelectricity, the study of the ferroelectric-to-paraelectric phase transition is central to the confirmation of the ferroelectric nature of a material, and great effort has been invested in establishing this transition in VDF-based copolymers. ${ }^{14,31-33}$ In addition, the operating 
temperature range is a key parameter for any candidate organic material for use in organic electronic devices. We measure the relevant temperature ranges of both the VDF oligomer source powder and the thin films using three difference methods. Because first-order phase transitions are highly sensitive to the rate of change in temperature, care was taken to steadily increase the temperature by $2{ }^{\circ} \mathrm{C} / \mathrm{min}$ in all three cases.

The heat flow per unit mass upon heating and cooling was collected via calorimetry for two consecutive cycles from the bulk powder VDF oligomer. The measured heat flow [Fig. 1(a)] shows a broad peak upon heating with the onset around $60^{\circ} \mathrm{C}$ and a maximum at $79 \pm 5.5^{\circ} \mathrm{C}$. This peak indicates that the VDF oligomer undergoes a ferroelectric-to-paraelectric phase transition at $79 \pm 5.5^{\circ} \mathrm{C}$ in the bulk, followed by a melting peak at $110 \pm 2{ }^{\circ} \mathrm{C}$. Upon cooling, the crystallization peak from the melt occurs at $100 \pm 1.5^{\circ} \mathrm{C}$ and the paraelectric-to-ferroelectric phase transition occurs at $45 \pm 1.5^{\circ} \mathrm{C}$.

Figure 1(b) shows the dependence of the pyroelectric current on substrate temperature measured for a glass/Pt $(40 \mathrm{~nm}) / \mathrm{VDF}(100 \mathrm{~nm}) / \mathrm{Al}(30 \mathrm{~nm})$ heterostructure with an electrode area of $200 \times 200 \mu \mathrm{m}^{2}$. The ferroelectric polarization was first saturated by applying incrementally increasing voltages across the film. Once the polarization was saturated, the pyroelectric current was recorded (using the Chynoweth method $^{29}$ ) as a function of substrate temperature upon heating. Consistent with previous measurements, ${ }^{25,27}$ the pyroelectric current increases with temperature and is a maximum at the proposed phase transition temperature. ${ }^{28}$ Upon cooling, the pyroelectric current was only $10 \%$ of the original saturated, room temperature value, indicating the depolarization of the VDF oligomer film upon heating, and is consistent with the expected behavior associated with heating above the phase transition temperature. From the pyroelectric measurement [Fig. 1(b)], the ferroelectric-toparaelectric phase transition temperature of the VDF thin film is found to be $65 \pm 1{ }^{\circ} \mathrm{C}$ on heating, $14{ }^{\circ} \mathrm{C}$ lower than the value found in the calorimetry measurements [Fig. 1(a)]. Understanding this difference may require a detailed comparative study of the thermo-kinetics of the phase transformations in these systems in both thin film and bulk forms. The present study does not address the thickness dependence of the ferroelectric transition temperature in these VDF oligomer thin films. However, this topic has been extensively studied in $\mathrm{P}(\mathrm{VDF}-\mathrm{TrFE})$ thin films, ${ }^{34}$ in which the transition temperature remains almost constant down to films only two monolayers thick.

Further evidence for the existence of the phase transition is given in Fig. 1(c), which shows the capacitance as a function of substrate temperature measured on a glass/Pt $(30 \mathrm{~nm}) /$ VDF $(200 \mathrm{~nm}) / \mathrm{Al}(30 \mathrm{~nm})$ heterostructure with an electrode area of $1.5 \times 1.5 \mathrm{~mm}^{2}$. The capacitance, which depends on the dielectric constant of the VDF oligomer, shows a clear peak at $100 \pm 1{ }^{\circ} \mathrm{C}$, indicating a phase transition. The $35^{\circ} \mathrm{C}$ difference in transition temperature of the films [Figs. 1(b) and $1(\mathrm{c})]$ is similar to that previously noted in $\mathrm{P}(\mathrm{VDF}-\mathrm{TrFE})$ thin films, ${ }^{34}$ in which pyroelectric and dielectric constant measurements upon heating indicated transition temperatures of 78 and $110^{\circ} \mathrm{C}$, respectively. The dielectric constant exhibits thermal hysteresis during a first-order phase transition, and thus, the capacitance peaks at a higher temperature upon heating than upon cooling. ${ }^{34}$ (It should be noted that the VDF oligomer film suffered melting related damage above the transition temperature, and thus we were not able to obtain meaningful cooling data.)

The $\mathrm{C}-\mathrm{V}$ and $\mathrm{I}-\mathrm{V}$ curves shown in Fig. 2 were measured on a glass/Pt $(30 \mathrm{~nm}) / \mathrm{VDF}(200 \mathrm{~nm}) / \mathrm{Al}(30 \mathrm{~nm})$ heterostructure with an electrode area of $1.5 \times 1.5 \mathrm{~mm}^{2}$. Figure 2 (a) shows $\mathrm{C}-\mathrm{V}$ loops for three consecutive cycles. The $\mathrm{C}-\mathrm{V}$ loops show the characteristic butterfly shape indicative of ferroelectricity, and the reproducibility of these C-V loops over three cycles reflects the stability of ferroelectric properties in these VDF oligomer thin films. Figure 2(b) shows the loss tangent as a function of bias voltage for the same sample, collected simultaneously with the capacitance loops shown in Fig. 2(a). The value of the loss tangent for these thermally evaporated films is comparable to solution-cast PVDF films $(0.06)^{35}$ and cluster synthesized VDF oligomer films $(0.07)^{36}$ at the same frequency used here $(1 \mathrm{kHz})$. When the ferroelectric polarization is saturated, the loss tangent is about $0.07-0.08$ and remains less than 0.11 even during polarization reversal. This low value indicates that the thermally evaporated VDF oligomer has a relatively low dielectric loss and is suitable for capacitor devices. Furthermore, the low dielectric loss of these VDF oligomer
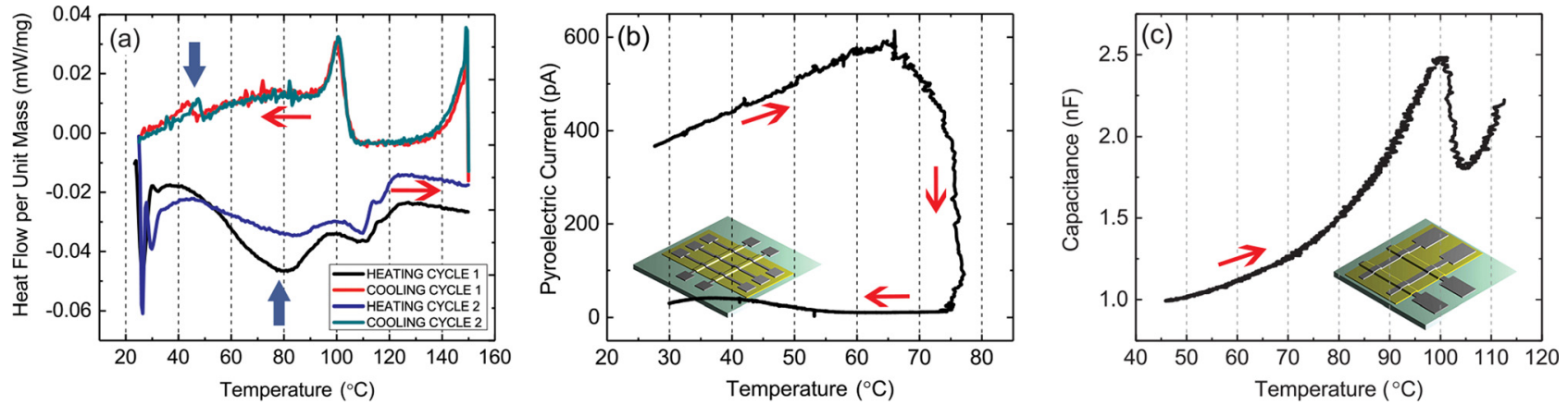

FIG. 1. (a) Heating (black and blue) and cooling (red and cyan) calorimetry measurements. The phase transitions are marked as blue arrows. (b) Pyroelectric current as a function of substrate temperature from a 100-nm thick VDF oligomer film. (c) Capacitance as a function of substrate temperature from a 200-nm thick VDF oligomer film. The red arrows in parts (a)-(c) indicate the direction of the curves, while the insets in parts (b) and (c) show the sample architecture for each measurement (the yellow layer is the VDF oligomer film). 

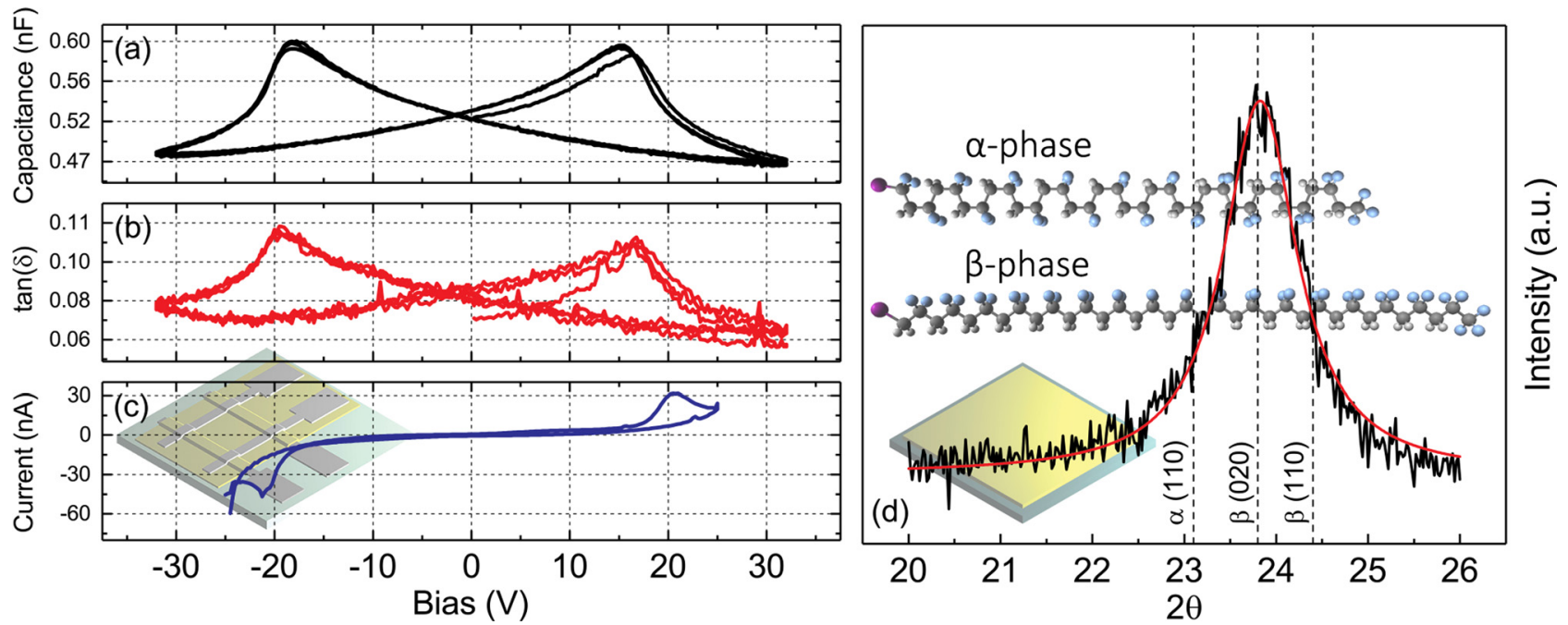

FIG. 2. (a) Capacitance and (b) loss tangent for three consecutive voltage loops, demonstrating the characteristic butterfly loops of ferroelectric materials, for a 200-nm thick VDF oligomer film. (c) Current as a function of voltage for a 200-nm thick VDF oligomer film. The inset in part (c) shows the sample architecture for the measurements in parts (a)-(c). (d) Background corrected XRD peak of an as-deposited VDF oligomer thin film (fit in red). The upper insets show illustrations of the $\alpha$-phase and $\beta$-phase chain conformations, while the lower inset shows the sample architecture for the measurement.

thin films is a strong indicator of the insulating nature of these films even across the fairly large area of $1.5 \times 1.5 \mathrm{~mm}^{2}$.

A typical I-V measurement from these heterostructures is shown in Fig. 2(c). At higher voltages, there is a nonlinear background due to conduction across the VDF oligomer. However, around $\pm 21 \mathrm{~V}$, a peak in the measured current can be seen and is due to the polarization reversal associated with the axial rotation of the VDF oligomer chains. The voltage was swept at a constant rate of $0.25 \mathrm{~V} / \mathrm{s}$. The time integral of the switching current, after correcting for the leakage current, divided by the area of the electrode yields a value of twice the remanent polarization, ${ }^{20}$ and so from Fig. 2(c), the remanent polarization is calculated to be $104 \pm 2 \mathrm{mC} / \mathrm{m}^{2}$. This value is less than the reported value of $130 \mathrm{mC} / \mathrm{m}^{2}$ by Noda et $a l .{ }^{20}$ but higher than that of the remanent polarization of PVDF $\left(60 \mathrm{mC} / \mathrm{m}^{2}\right)^{16}$ and comparable to that of $\mathrm{P}(\mathrm{VDF}-\mathrm{TrFE})\left(100 \mathrm{mC} / \mathrm{m}^{2}\right) .{ }^{37}$ Although the value for remanent polarization found here is lower than that reported in Ref. 20, we note that the chain length of the VDF oligomer in that study differs from that used here. Previous studies have shown that the chain length can affect the crystal structure of VDF oligomer thin films, ${ }^{38}$ which in turn can affect the measured polarization.

While $\mathrm{C}-\mathrm{V}$ and $\mathrm{I}-\mathrm{V}$ loops demonstrate the switchable polarization of the thin films, XRD can be used to determine the chain conformation and dipole orientation of these thermally evaporated, large area VDF oligomer films. Figure 2(d) shows the XRD peak of a 100-nm thick VDF oligomer film deposited on a $\mathrm{Si}$ wafer. A Lorentz peak fit indicates a peak location of $23.81^{\circ}$, clearly indicating that the dominant crystalline orientation and chain conformation of the as-grown VDF oligomer film are the (020) orientation of the $\beta$-phase, ideal for device applications, with the maximum ferroelectric polarization perpendicular to the sample plane. ${ }^{25}$ In contrast, the dipole moments of the (110) orientation of the $\beta$-phase (which is the usual orientation for Langmuir-Blodgett deposited films of $\mathrm{P}(\mathrm{VDF}-\mathrm{TrFE})^{5,39}$ are canted $30^{\circ}$ away from the surface normal, resulting in a $13.4 \%$ decrease in the out-ofplane component of ferroelectric polarization. The LangmuirBlodgett deposition of the $\beta$-phase VDF oligomer results in films with the carbon chains normal to the substrate, such that the dipole moments are oriented in the plane of the film, ${ }^{40,41}$ an orientation that is both difficult to measure and less useful in device applications.

The hysteretic behavior in Figs. 2(a)-2(c) and the location of the diffraction peak in Fig. 2(d) demonstrate the consistently switchable polarization and optimal crystalline orientation of the as-grown VDF oligomer, respectively. Moreover, the large electrode areas of $2.25 \mathrm{~mm}^{2}$ compare very favorably with those needed by organic-based electronic devices, such as an OLED pixel (on the order of $\mu \mathrm{m}^{2}$ ) or a ferroelectric RAM bit (on the order of $\mathrm{nm}^{2}$ ), and indicate that the films can be deposited in large, defect free areas suitable for device production.

An additional measure of the suitability of ferroelectrics for use in non-volatile devices is the long term stability of the ferroelectric polarization in the absence of an electric field. Figure 3(a) shows a typical pyroelectric hysteresis loop collected via the Chynoweth method from a glass $/ \mathrm{Pt}(50 \mathrm{~nm}) / \mathrm{Co}$ $(1 \mathrm{~nm}) / \mathrm{VDF}(175 \mathrm{~nm}) / \mathrm{Al}(30 \mathrm{~nm})$ heterostructure with an electrode area of $200 \times 200 \mu \mathrm{m}^{2}$. The red arrows indicate the path of the hysteresis. At a constant temperature, the pyroelectric current is directly proportional to the ferroelectric polarization of the VDF oligomer thin film, and thus, a saturated pyroelectric current is equivalent to complete polarization. The initial measured pyroelectric current, prior to the application of an external voltage, is $5 \mathrm{pA}$, less than $1 \%$ of the saturation current of $650 \mathrm{pA}$. Additional hysteresis loops collected from other metal/oligomer heterostructures repeatedly show this small initial pyroelectric current, ranging from $1 \%$ to $10 \%$ of the saturation current, indicating that the net polarization of the as-grown, unpoled VDF oligomer thin films is low.

The process of obtaining the hysteresis loop shown in Fig. 3(a) was as follows. The polarization state was prepared 

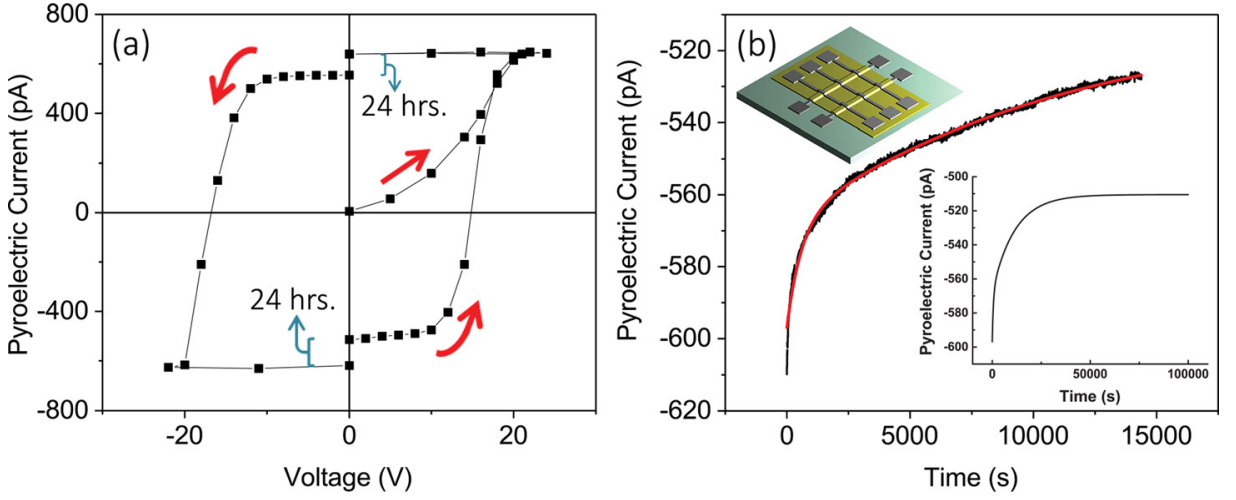

FIG. 3. (a) Hysteresis in the pyroelectric response from a $175-\mathrm{nm}$ thick VDF oligomer film. The film was left for $24 \mathrm{~h}$ at each remanent state. The red arrows indicate the direction of the hysteresis loop. (b) Pyroelectric current as a function of time at the negative remanent state (i.e., at zero voltage). An exponential fit is shown in red. The lower inset shows the fitted function on the scale of $24 \mathrm{~h}$. The upper inset shows the sample architecture for these measurements. by applying a constant voltage held across the VDF oligomer thin film for five minutes, after which the voltage is disconnected and the current is measured for several seconds using a lock-in amplifier with a time constant of $300 \mathrm{~ms}$. Once the pyroelectric current is recorded, the next incremental voltage is applied and the process continues until the entire hysteresis loop is obtained. ${ }^{29,42}$ For the loop shown, the exception to this procedure occurs at the positive and negative remanent polarization states. At these locations along the hysteresis loop, the pyroelectric current was recorded shortly after the application of voltage as usual. The sample was then left in the remanent state, with no applied voltage, for $24 \mathrm{~h}$, after which the pyroelectric current was recorded again and collection of the hysteresis loop proceeded once more. The pyroelectric signal decayed by $13 \%$ (17\%) after $24 \mathrm{~h}$ at the positive (negative) remanent saturation state. The decay is interpreted as a gradual relaxation of the VDF oligomer chains back towards the as-grown low net polarization state, decreasing the net ferroelectric polarization perpendicular to the metal electrodes. Since the as-grown VDF oligomer film is predominantly in the $(020)$ orientation [Fig. 2(d)], this zero-field decay is most likely due to the film breaking up into up and down domains.

The use of a non-volatile device makes it imperative that the VDF oligomer film retains a non-zero remanent polarization. Polarization relaxation in Langmuir-Blodgett films of P(VDF-TrFE) has been studied by monitoring the pyroelectric current as a function of time, ${ }^{43}$ and the same approach is used here. The time dependence of the pyroelectric current over a period of over four hours was measured and is shown in Fig. 3(b), where $t=0 \mathrm{~s}$ corresponds to the current immediately after reaching the negative remanent polarization state. The red line is an exponential fit to the decaying pyroelectric signal. From the fit, the decaying signal is found to have a time constant of $6700 \mathrm{~s}$. The inset of Fig. 3(b) shows the fitted function on a much longer time scale of about $24 \mathrm{~h}$. The fit shows very little additional decay after $12 \mathrm{~h}$, indicating that the small jump in the hysteresis loop shown in Fig. 3(a) represents most of the polarization decay at the remanent states. Extrapolation to $48 \mathrm{~h}$ shows an additional decay of less than $1 \%$. Indeed, a non-zero pyroelectric current can be measured on samples after having been left in the remanent polarization state for several months. From these data, we conclude that even after an extended period of time, a significant portion of the remanent polarization signal remains, in this case about $85 \%$, and nearly all of the decay occurs within the first $12 \mathrm{~h}$. This nonzero, stable remanent polarization is a necessary condition for any potential non-volatile device applications.

Figure 4 shows PFM measurements with a spatial resolution of $30 \mathrm{~nm}$ collected on various $\mathrm{Si} / \mathrm{Pt}(30 \mathrm{~nm}) / \mathrm{VDF}$ $(50 \mathrm{~nm})$ samples. The $\mathrm{Si} / \mathrm{Pt}$ substrate serves as a bottom electrode, while the PFM tip itself serves as the top electrode, through which voltages are applied across the VDF oligomer thin film. Initially, a $12 \mu \mathrm{m} \times 12 \mu \mathrm{m}$ area was imaged with ac voltage $\left(\mathrm{V}_{\mathrm{dc}}=0 \mathrm{~V}\right)$ to detect any as-grown domains [Fig. 4(a)]. No domain structures were visible, indicating that the as-grown VDF oligomer films are nominally unpoled at the scale of the instrument resolution, in agreement with the low pyroelectric current of the as-grown film shown in Fig. 3(a). To demonstrate domain reading/writing, a square domain of $8 \mu \mathrm{m} \times 8 \mu \mathrm{m}$ within the same region imaged in Fig. 4(a) was written by applying a $-10 \mathrm{~V}$ bias across the VDF oligomer thin film [Fig. 4(b)]. After writing, the created domains were read via ac voltage with the dc bias off, revealing an increase in the piezoresponse amplitude in the poled area and a sharp phase contrast. A similar poling process albeit with $+10 \mathrm{~V}$ across the VDF oligomer thin film in the same region yields similar results as shown in Fig. 4(c). The choice of the dc writing voltage of $\pm 10 \mathrm{~V}$ was based on our past work, ${ }^{25}$ indicating that the average coercive field of these VDF oligomer thin films is $95 \mathrm{MV} / \mathrm{m}$. Hence, a bias of $\pm 10 \mathrm{~V}$ is sufficient to saturate the polarization in the $50 \mathrm{~nm}$ thick VDF oligomer film, which should have a switching voltage of about $\pm 5 \mathrm{~V}$.

In order to compare the piezoresponse amplitude of the positively and negatively poled regions, an $8 \mu \mathrm{m} \times 8 \mu \mathrm{m}$ area was first poled with $-10 \mathrm{~V}$ dc bias, followed by a smaller $4 \mu \mathrm{m} \times 4 \mu \mathrm{m}$ area, within the negatively poled region, written with $+10 \mathrm{~V}$ dc bias [Fig. 4(d)]. The resulting domain pattern revealed a high piezoresponse amplitude signal in the entire region with a $180^{\circ}$ phase contrast between the out-of-plane poled, antiparallel domains created by the $\pm 10 \mathrm{~V}$ dc bias. These PFM measurements clearly show the robust quality of the VDF oligomer films and the creation, erasure, and switching of locally created ferroelectric domains. To ensure that the piezoresponse amplitude and phase contrast shown in Figs. 4(b)-4(d) are in fact due to a ferroelectric response, local piezoresponse hysteresis measurements were also performed on similar $\mathrm{Si} / \mathrm{Pt} / \mathrm{VDF}$ oligomer heterostructures. 

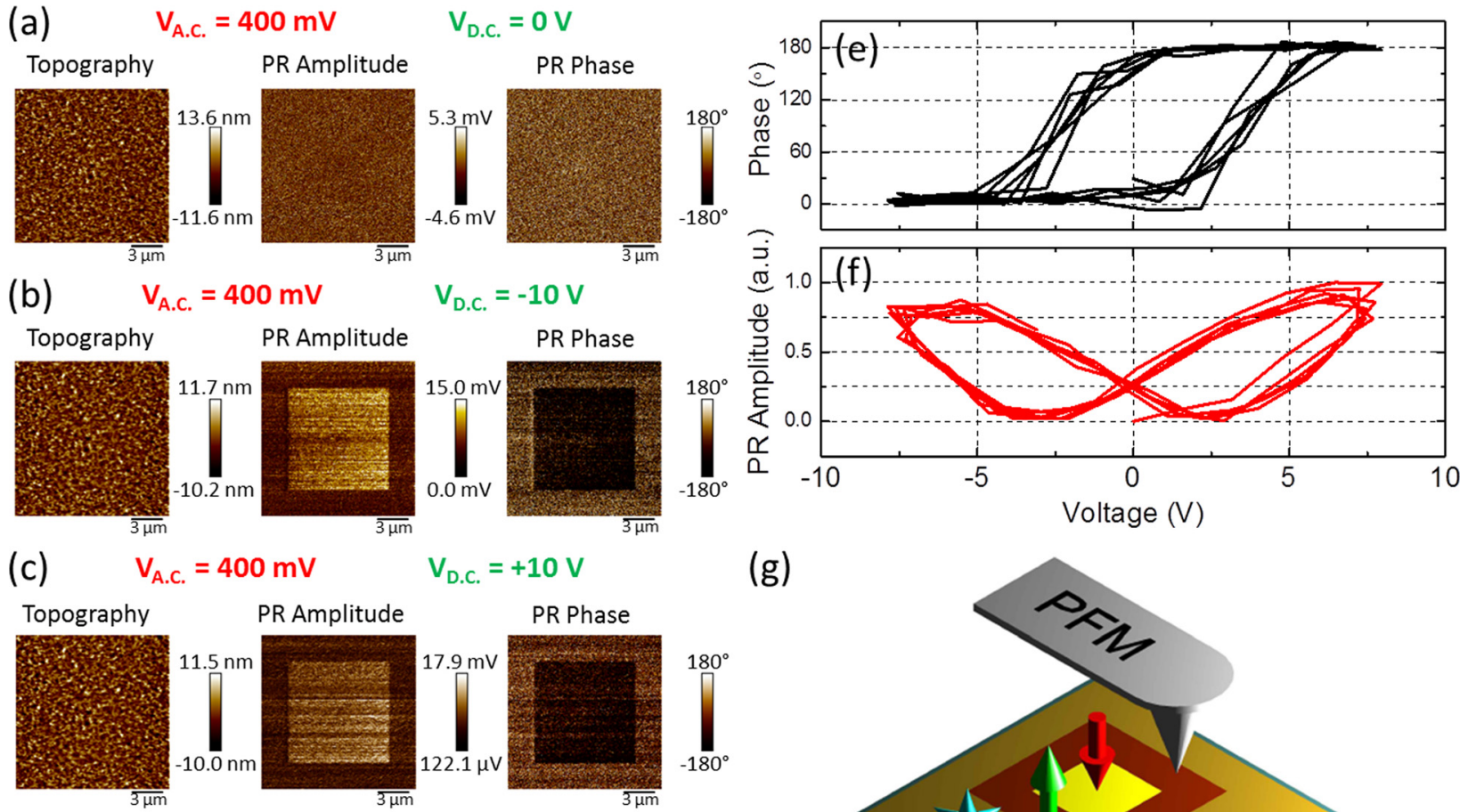

(g)

(d) $\quad V_{\text {A.c. }}=550 \mathrm{mV}$

$\mathrm{V}_{\text {D.c. }}= \pm 10 \mathrm{~V}$
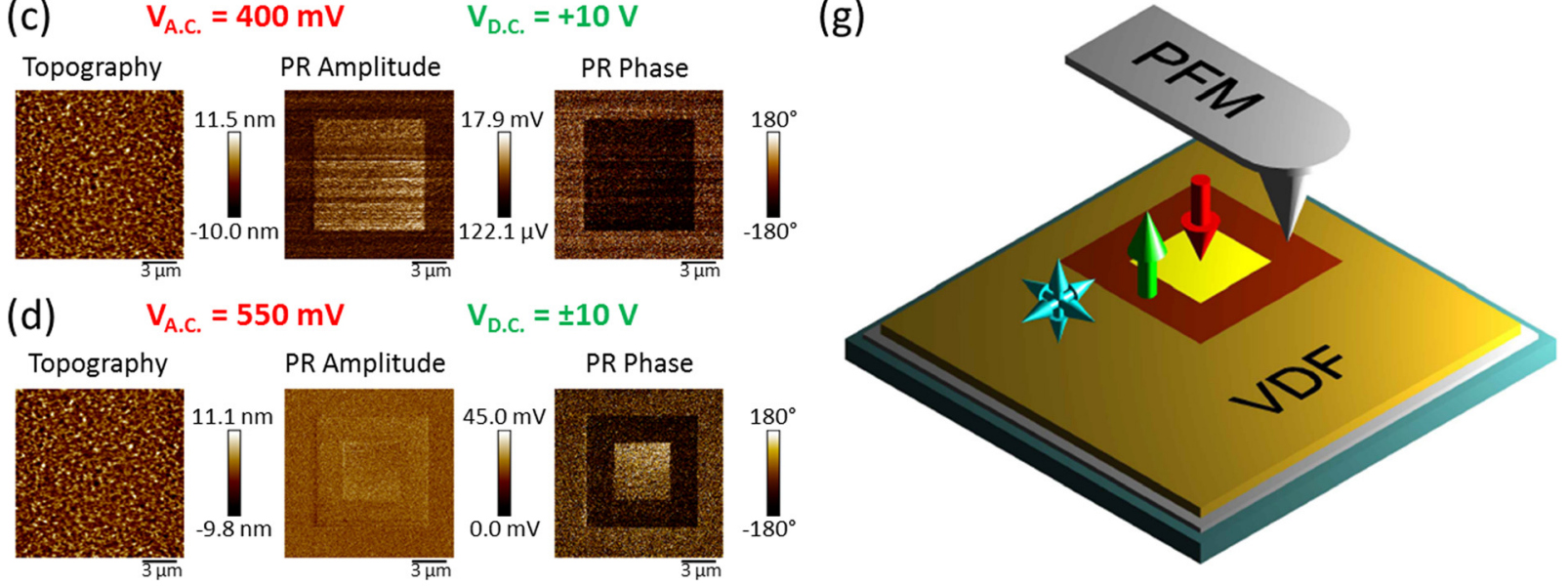

FIG. 4. (a)-(d) PFM measurements of topography (left), piezoelectric response (PR) amplitude (center), and PR phase (right) from VDF oligomer thin films under various ac (red) and dc (green) bias conditions. All scale bars are $3.0 \mu \mathrm{m}$. (e) and (f) Local (e) PR phase and (f) PR amplitude demonstrating clear hysteretic behavior. (g) An illustration of ferroelectric domain writing with a PFM tip, as demonstrated in part (d). The ferroelectric polarization of the poled VDF oligomer is represented by the red and green arrows, while the blue arrows represent the unpolarized, as-grown VDF oligomer.

Figs. 4(e) and 4(f) show the phase and piezoresponse amplitude from such a measurement, clearly demonstrating classic ferroelectric hysteresis. Figures 3(a), 4(e), and 4(f) demonstrate the switchable, ferroelectric behavior of the VDF oligomer on both macro- and microscopic scales.

Recall from Fig. 3 that the ferroelectric polarization was completely saturated before recording the time dependence of the polarization decay. Therefore, the data in Fig. 3 essentially correspond to a ferroelectric domain with an area of $200 \times 200 \mu \mathrm{m}^{2}$. Although the domains shown in Fig. 4 are smaller than $200 \times 200 \mu \mathrm{m}^{2}$, they are still on the order of $\mu \mathrm{m}^{2}$ and far larger than the critical domain size due to the depolarization field (on the order of $\mathrm{nm}^{2}$ ). ${ }^{34,44}$ Therefore, it is expected that the domains shown in Fig. 4 should have similar temporal stability as those corresponding to the data shown in Fig. 3.

\section{CONCLUSIONS}

This work confirms ferroelectricity in VDF oligomer thin films and more importantly establishes the existence of the ferroelectric-to-paraelectric phase transition. Calorimetry, pyroelectric, and capacitance measurements all clearly show a phase transition in both bulk powder and thin films on heating.
$\mathrm{C}-\mathrm{V}$ and I-V measurements clearly show the ferroelectric stability of these thermally evaporated VDF oligomer thin films. The ferroelectric polarization of these films can be repeatedly switched with low leakage. The spontaneous polarization of these VDF oligomer thin films is found to be $104 \pm 2 \mathrm{mC} / \mathrm{m}^{2}$, larger than that for PVDF and close to the value for P(VDFTrFE). The remanent polarization is relatively stable, relaxing $13 \%-17 \%$ in $12 \mathrm{~h}$ and relaxing more slowly after that. XRD measurements indicate that the as-grown state of these allvacuum deposited VDF oligomer thin films are deposited in the ferroelectric $\beta$-phase with the optimal (020) crystalline orientation. Thus, not only the interfacial cleanliness is maintained by the vacuum deposition but also the out-of-plane component of the ferroelectric polarization is maximized, and hence, these films do not require post deposition processing. Finally, PFM measurements show that ferroelectric domains can be written in VDF oligomer thin films and that the phase contrast between antiparallel, out-of-plane domains is high.

In summary, we establish the viability of thermally evaporated VDF oligomer thin films for use in a variety of applications and devices. The spontaneous polarization of the VDF oligomer rivals that of $\mathrm{P}(\mathrm{VDF}-\mathrm{TrFE})$, but the oligomer can be deposited in vacuum, preserving interfacial cleanliness. Furthermore, these measurements were made on 
large area samples, indicating that the superior quality of the thermally evaporated VDF oligomer films can be maintained across areas suitable for devices. The stable remanent polarization and excellent domain contrast open channels for nonvolatile memory applications in particular, thus establishing the VDF oligomer as a strong candidate material in the rapidly developing field of organic electronics.

\section{ACKNOWLEDGMENTS}

Financial support was provided by the National Science Foundation (NSF) through the Nebraska Materials Research Science and Engineering Center (MRSEC) under Grant No. DMR-1420645 and by NSF Grant No. ECCS-1101256. This research was performed in part in the Nebraska Nanoscale Facility: National Nanotechnology Coordinated Infrastructure and the Nebraska Center for Materials and Nanoscience, which are supported by the National Science Foundation under Award No. ECCS-1542182 and the Nebraska Research Initiative.

${ }^{1}$ J. Hwang, A. Wan, and A. Kahn, Mater. Sci. Eng.: R: Rep. 64, 1 (2009).

${ }^{2}$ G. Gu, V. Bulović, P. E. Burrows, S. R. Forrest, and M. E. Thompson, Appl. Phys. Lett. 68, 2606 (1996).

${ }^{3}$ C. Hochfilzer, G. Leising, Y. Gao, E. Forsythe, and C. W. Tang, Appl. Phys. Lett. 73, 2254 (1998).

${ }^{4}$ S. Tokito, T. Tsutsui, and Y. Taga, J. Appl. Phys. 86, 2407 (1999).

${ }^{5}$ J. Song, H. Lu, K. Foreman, S. Li, L. Tan, S. Adenwalla, A. Gruverman, and S. Ducharme, J. Mater. Chem. C 4, 5914 (2016).

${ }^{6}$ K. Fujita, T. Yasuda, and T. Tsutsui, Appl. Phys. Lett. 82, 4373 (2003).

${ }^{7}$ F. Eder, H. Klauk, M. Halik, U. Zschieschang, G. Schmid, and C. Dehm, Appl. Phys. Lett. 84, 2673 (2004).

${ }^{8}$ A. Mardana, S. Ducharme, and S. Adenwalla, Nano Lett. 11, 3862 (2011).

${ }^{9}$ P. V. Lukashev, T. R. Paudel, J. M. López-Encarnación, S. Adenwalla, E. Y. Tsymbal, and J. P. Velev, ACS Nano 6, 9745 (2012).

${ }^{10}$ R.-Q. Wang, W.-J. Zhu, H.-C. Ding, S.-J. Gong, and C.-G. Duan, J. Appl. Phys. 115, 043909 (2014)

${ }^{11}$ L. E. Cross, Mater. Chem. Phys. 43, 108 (1996).

${ }^{12}$ M. Ye. Zhuravlev, R. F. Sabirianov, S. S. Jaswal, and E. Y. Tsymbal, Phys. Rev. Lett. 94, 246802 (2005).

${ }^{13}$ A. Q. Jiang, C. Wang, K. J. Jin, X. B. Liu, J. F. Scott, C. S. Hwang, T. A. Tang, H. B. Lu, and G. Z. Yang, Adv. Mater. 23, 1277 (2011).

${ }^{14}$ T. Furukawa, Phase Transitions 18, 143 (1989).

${ }^{15}$ Y. Pei and X. C. Zeng, J. Appl. Phys. 109, 093514 (2011).

${ }^{16}$ T. Furukawa, M. Date, and E. Fukada, J. Appl. Phys. 51, 1135 (1980).
${ }^{17}$ K. Tashiro, Ferroelectric Polymers: Chemistry, Physics, and Applications, edited by H. S. Nalwa (Marcel Dekker, Inc., New York, 1995).

${ }^{18}$ M. Poulsen and S. Ducharme, IEEE Trans. Dielectr. Electr. Insul. 17, 1028 (2010)

${ }^{19}$ K. Noda, K. Ishida, T. Horiuchi, K. Matsushige, and A. Kubono, J. Appl. Phys. 86, 3688 (1999).

${ }^{20}$ K. Noda, K. Ishida, A. Kubono, T. Horiuchi, H. Yamada, and K. Matsushige, J. Appl. Phys. 93, 2866 (2003).

${ }^{21}$ S. Palto, L. Blinov, A. Bune, E. Dubovik, V. Fridkin, N. Petukhova, K. Verkhovskaya, and S. Yudin, Ferroelectr. Lett. 19, 65 (1995).

${ }^{22}$ S. J. Kang, Y. J. Park, J. Sung, P. S. Jo, C. Park, K. J. Kim, and B. O. Cho, Appl. Phys. Lett. 92, 012921 (2008).

${ }^{23}$ K. Foreman, E. Echeverria, M. A. Koten, R. M. Lindsay, N. Hong, J. Shield, and S. Adenwalla, Mater. Res. Express 3, 116403 (2016).

${ }^{24}$ K. Foreman, C. Labedz, M. Shearer, and S. Adenwalla, Rev. Sci. Instrum. 85, 043902 (2014).

${ }^{25}$ K. Foreman, N. Hong, C. Labedz, M. Shearer, S. Ducharme, and S. Adenwalla, J. Phys. D: Appl. Phys. 49, 015301 (2016).

${ }^{26}$ A. Takeno, N. Okui, T. Kitoh, M. Muraoka, S. Umemoto, and T. Sakai, Thin Solid Films 202, 205 (1991).

${ }^{27}$ A. V. Bune, C. Zhu, S. Ducharme, L. M. Blinov, V. M. Fridkin, S. P. Palto, N. G. Petukhova, and S. G. Yudin, J. Appl. Phys. 85, 7869 (1999).

${ }^{28}$ M. E. Lines and A. M. Glass, Principles and Applications of Ferroelectrics and Related Materials (Clarendon, Oxford, 1977).

${ }^{29}$ A. G. Chynoweth, J. Appl. Phys. 27, 78 (1956).

${ }^{30}$ C. A. Schneider, W. S. Rasband, and K. W. Eliceiri, Nat. Methods 9, 671 (2012).

${ }^{31}$ T. Furukawa, G. E. Johnson, H. E. Bair, Y. Tajitsu, A. Chiba, and E. Fukada, Ferroelectrics 32, 61 (1981).

${ }^{32}$ T. Furukawa, Ferroelectrics 57, 63 (1984).

${ }^{33}$ R. G. Kepler and R. A. Anderson, Adv. Phys. 41, 1 (1992).

${ }^{34}$ A. V. Bune, V. M. Fridkin, S. Ducharme, L. M. Blinov, S. P. Palto, A. V. Sorokin, S. G. Yudin, and A. Zlatkin, Nature 391, 874 (1998).

${ }^{35}$ L. L. Sun, B. Li, Y. Zhao, G. Mitchell, and W. H. Zhong, Nanotechnology 21, 305702 (2010)

${ }^{36}$ B. Balasubramanian, K. L. Kraemer, S. R. Valloppilly, S. Ducharme, and D. J. Sellmyer, Nanotechnology 22, 405605 (2011).

${ }^{37}$ H. Ohigashi, K. Omote, and T. Gomyo, Appl. Phys. Lett. 66, 3281 (1995).

${ }^{38}$ Herman, S. Umemoto, T. Kikutani, and N. Okui, Polym. J. 30, 659 (1998).

${ }^{39}$ M. Bai and S. Ducharme, Appl. Phys. Lett. 85, 3528 (2004).

${ }^{40}$ R. Korlacki, J. T. Johnson, J. Kim, S. Ducharme, D. W. Thompson, V. M. Fridkin, Z. Ge, and J. M. Takacs, J. Chem. Phys. 129, 064704 (2008).

${ }^{41}$ P. Sharma, S. Poddar, R. Korlacki, S. Ducharme, and A. Gruverman, Appl. Phys. Lett. 105, 022906 (2014).

${ }^{42}$ A. Bune, S. Ducharme, V. Fridkin, L. Blinov, S. Palto, N. Petukhova, and S. Yudin, Appl. Phys. Lett. 67, 3975 (1995).

${ }^{43}$ A. V. Sorokin, V. M. Fridkin, and S. Ducharme, J. Appl. Phys. 98, 044107 (2005).

${ }^{44}$ J. Junquera and P. Ghosez, Nature 422, 506 (2003). 